\title{
An XHV atom probe with ultra-low hydrogen background
}

Peter Felfer, Benedict Ott, Martina Heller and Chandra Macauley

FAU Erlangen, Erlangen, United States

The time-of-flight mass spectrometry principle that is used in atom probe tomography fundamentally allows for the detection of all chemical elements across the periodic table. However, so far $\mathrm{H}$ has been difficult to detect, as the $\mathrm{H}$ that was originally present in the sample is occluded by $\mathrm{H}$ that is emitted by the stainless steel of the UHV system [1]. A workaround has been to dope materials with deuterium $\left({ }^{2} \mathrm{H}\right.$, D) instead, which has a low natural abundance [2,3]. D however can only be used in experiments where small amounts of material are used, due to cost and availability.

In order to be able to quantify $\mathrm{H}$ in materials without the need for $\mathrm{D}$, or to use $\mathrm{D}$ as a tracer, the author has built an atom probe that does not use steel, but rather titanium as the main material for the main chamber. $\mathrm{Ti}$ is known to result in vacuum systems with ultra-low $\mathrm{H}$ background [4], while otherwise being a dropin replacement for steel.

This instrument is built around a commercially available delayline detector with funnel type microchannel plates. This detector has in-line pulse processing in the TDC, which sends the hit coordinates and flight times to a control PC via USB 3.0. A meander type delayline is used to minimize crosstalk from the high voltage pulse. The high voltage pulse is created by a drift-step recovery diode pulser with a maximum amplitude of $3.5 \mathrm{kV}$ and repetition rate of $200 \mathrm{kHz}$ into a well-shielded and impedance matched exchangeable counterelectrode. A piezo stage is used to align the samples with respect to the counterelectrode. An actively cooled specimen carousel in the buffer chamber allows for the sampels to be stored at cryogenic temperatures. The samples can be inserted through either a "cryo load lock", which interfaces to a cryo transfer shuttle, or through a regular load lock, which can be heated to "bake" new counterlectrodes. In this talk the author will present results on the performance of this instrument, with a special focus on the ability to unambiguously detect ${ }^{1} \mathrm{H}$ in materials.

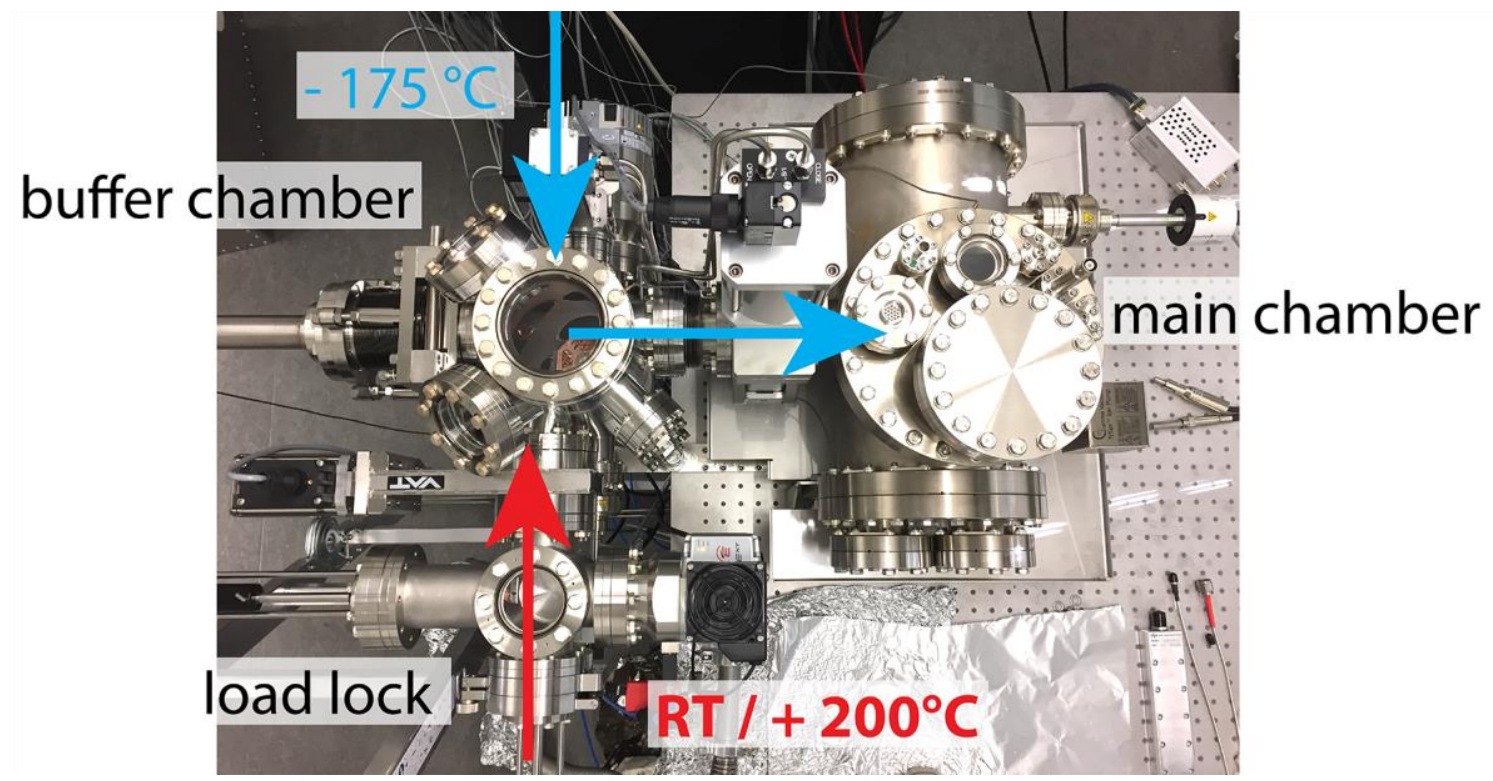

Figure 1. Overview of the atom probe instrument, showing cold and hot specimen insertion routes 
[1] P. A. Redhead: Extreme high vacuum, CERN Report No 99-05, 213 (1999).

[2] J. Takahashi et al., The first direct observation of hydrogen trapping sites in TiC precipitationhardening steel through atom probe tomography, Scripta Materialia 63 (2010), 261-264.

[3] Y.-S. Chen et al., Direct observation of individual hydrogen atoms at trapping sites in a ferritic steel, Science 355 (2017), 1196-1199.

[4] H Kurisu et al., Application of Titanium Materials to Vacuum Chambers and Components, Journal of Physics: Conference Series 100 (2008) 